\title{
Estrategias para propiciar la propincuidad digital en la adquisición de ELE en contextos de brecha digital
}

\author{
Strategies conducive to digital propinquity when acquiring
}

Spanish as a foreign language in a digitally divided context

\author{
NeUS GIMENO GIMENO \\ LECTORA AECID-MAEC eN LA UNIVERSIDAD JaWAHARLAL NeHRU \\ neusgigi@gmail.com
}

\begin{abstract}
Resumen
La pandemia de la COVID-19 ha agravado la equidad en el acceso a la tecnología de muchos estudiantes. Como consecuencia, ha habido un gran ausentismo estudiantil y el concepto de comunidad en el aula se ha visto menoscabado. De ahí que la interacción en la adquisición de lenguas en general $y$, concretamente, del español como lengua extranjera se haya reducido drásticamente pese a ser uno de los elementos clave en esta disciplina. Por ello, se presentan estrategias que favorecen la propincuidad digital para crear comunidades de aprendizaje vivas y cambiantes y, a su vez, fomentar paralelamente la independencia cognitiva y la interdependencia social desde el modelo de "Comunidad de indagación".

Palabras clave: español como lengua extranjera, brecha digital, propincuidad digital, comunidad de indagación, interacción
\end{abstract}

\begin{abstract}
The COVID-19 pandemic has accentuated a lack of access to technology among many students. This has been one of the main reasons for student absenteeism, which in turn has diminished the sense of community in the classroom. Consequently, interaction in language acquisition, specifically Spanish as a foreign language, has grown smaller, despite it being one of the key elements within this discipline. In order to facilitate access to digital learning, strategies that enhance digital propinquity so that active, reactive and evolving communities are presented in this study. This, in turn, not only boosts cognitive independence but it also generates social interdependence from the "Community of Inquiry" perspective.
\end{abstract}

Key words: Spanish as a foreign language, digital divide, digital propinquity, flipped classroom, community of inquire, interaction 


\section{Introducción}

Después de que la OMS declarara la pandemia por la COVID-19 el 11 de marzo de 2020, en la mayoría de países se limitó la movilidad de las personas y se impuso una separación física en el ámbito social y profesional. En semejantes condiciones, las universidades se vieron obligadas a suspender toda la actividad académica en formato presencial y a continuarla en la modalidad digital. Esta celeridad con la que las instituciones educativas tuvieron que implementar una transición tan precipitada -que Hodges et al. (2020) han llamado "enseñanza remota de emergencia" - evidenció los déficits estructurales de la misma: la falta de equidad, tanto en el acceso (también llamada "brecha digital"), como en el progreso de la trayectoria académica (Ariño et al., 2019).

Esto es de especial relevancia en el caso de la adquisición del español como lengua extranjera ( $y$ de otras lenguas), ya que la interacción, como se detalla más adelante, juega un papel central en esta disciplina y se vio gravemente afectada por los daños colaterales de esta enseñanza remota de emergencia (conexión a internet inestable o insuficiente, falta de motivación, estrés psicológico entre otros). Como consecuencia, se redujo el aprendizaje interactivo entre los estudiantes y el sentimiento de comunidad entre ellos.

Dadas estas circunstancias, fue ineludible adoptar estrategias insólitas para hacer frente a esta casuística tan inaudita. En primer lugar, en este trabajo se propone una reorganización de la carga de trabajo, en concreto de la enseñanza síncrona y asíncrona, dándole a esta última mayor peso por su ubicuidad y flexibilidad. En segundo lugar, se presentan estrategias para alentar el aprendizaje interactivo entre estudiantes que, a su vez, fomentan la propincuidad o cercanía - si bien digital- desde la perspectiva del modelo de "Comunidad de Indagación" (detallado más adelante).

Esto es de especial relevancia en contextos donde hay una profunda brecha digital (en este caso, se toma como ejemplo una universidad pública de India). Por tanto, aunque esta reflexión nació en un momento histórico de crisis, se puede extrapolar a otros contextos similares.

\section{Sobre el concepto de "interacción"}

La interacción desempeña un papel crucial en el proceso de aprendizaje de una segunda lengua (Mackey y Polio 2009). Esta puede ser definida como "el intercambio y la negociación del sentido entre dos o más participantes situados en contextos sociales" (O'Sullivan, et al., 1997: 196). También puede ser entendida como un tipo de actividad comunicativa realizada por dos o más participantes que se influyen mutuamente, en un intercambio de acciones y reacciones verbales y no verbales (García-Valcárcel, et al., 2012) ${ }^{1}$.

\footnotetext{
${ }^{1}$ El Marco Común Europeo de Referencia para las lenguas (MCER), puesto que la interacción una de las cuatro actividades de la lengua junto a la comprensión, la expresión y la
} 
La interacción en al aula ha jugado un papel central en la enseñanza y adquisición de lenguas extranjeras en la era de la enseñanza comunicativa (Ellis, 1991; Krashen, 1989; Larsen-Freeman \& Long, 1991; Mackey, Abbuhl, \& Gass, 2012; Swain, 2000). También ha habido muchos docentes, investigadores y expertos que han estudiado al detalle el discurso del docente, la respuesta del aprendiente y la retroalimentación, conocido también como "discurso del aula" (Liskinasih, 2016; Maolida, 2013; Roostini, 2011; Walsh, 2011).

En el campo de la adquisición de segundas lenguas, la negociación del significado a través de inputs modificados ocurre en una conversación interaccional, es decir, aquella que se emplea para desarrollar y mantener una relación social o para la expresión de uno mismo (G. Brown y G. Yule, 1983). A través de la negociación conversacional y el ajuste lingüístico se obtiene un input que está integrado en la lengua adquirida (Mayo \& Soler, 2013). No obstante, el input comprensible no es suficiente a menos que el aprendiente tenga numerosas oportunidades para usar esta lengua y producir mensajes que sean comprensibles - outputs- y, en lo posible, correctos y adecuados al contexto conversacional (Swain 1985; Lantolf 2006, Mackey et al., 2012). Según la tarea y el contexto de comunicación, en la interacción podrán surgir secuencias de negociación del significado (Long 1985; Jauregi 1997), así como de la forma (Doughty y Long 1998; Long y Robinson 1998), clave en el proceso de aprendizaje. También cabe destacar la relevancia que tienen el uso de la lengua meta en un contexto social y auténtico (Ellis 2003; Lantolf 2006). Por ello, es indispensable que el aprendiente sea capaz de interpretar y crear significados adecuadamente en un evento comunicativo propio de los representantes de diferentes comunidades lingüísticas y culturales Byram 1997; Kasper 2001).

Como Thoms (2012) resumió, desde un punto de vista teórico sociocultural, los aspectos más importantes de una lengua están relacionados y han sido formados por las estrategias en las que las personas interactúan con el resto de ellas en diferentes contextos comunicativos. Además, los aprendientes de una lengua desarrollan sus competencias en interacciones y relaciones sociales al participar en la comunicación con otros participantes con más experiencia, conocimiento y competencia, tales como el docente y/o otros compañeros. De ahí que se pueda demostrar la relevancia de la interacción grupal en la adquisición del español como lengua extranjera.

\section{El impacto de la pandemia la COVID-19 en la educación: el aprendizaje en línea}

Debido a la crisis sanitaria ocasionada por la rápida propagación de la COVID19 y a la imposición del confinamiento por parte de la mayoría de los

mediación, le otorga un papel central en la comunicación, ya que los usuarios de la lengua alternan sus roles de hablante y oyente para construir, conjuntamente, una conversación mediante la negociación de significados siguiendo el principio de cooperación (MCER 88). 
gobiernos en marzo de 2020, se cerraron los centros educativos y hubo una transición inesperada y brusca de una enseñanza presencial a una digital que afectó al 90\% de estudiantes de los matriculados en 194 países².

Esta suspensión preventiva de la educación presencial alteró radicalmente los planes educativos en muchos países, provocando la transición hacia la enseñanza a distancia de emergencia: forzosa, sin transición ni previsión (formación, competencia, recursos, etc.), algo que puso de relieve la inexorable presencialidad de las estructuras organizativas, así como las dificultades para acceder a la enseñanza en línea por parte de muchos estudiantes y la complejidad de la inclusión y de la atención a la diversidad en un contexto virtual ${ }^{3}$.

En el caso de las universidades, en las que siempre se había desarrollado la actividad educativa bajo el paradigma presencial, el salto al marco online supuso un cambio sustancial en la organización, que generó importante rechazo (García-Peñalvo, 2020).

En este contexto tan único, es importante ver el aprendizaje no como un proceso de transmisión de información, sino como uno social y cognitivo (Hodges, Moore, Lockee, Trust y Bond, 2020). Por eso, a la hora de planificar la enseñanza en línea, es necesario también tener presentes las diferentes interacciones que ocurren en el proceso y cómo aumentan los resultados de aprendizaje (Bernard et al., 2009).

El cierre de los centros educativos y la adopción de la enseñanza a distancia afectó negativamente los procesos cognitivos de los estudiantes por diferentes motivos: el estrés, un cambio en la forma en que los estudiantes interactúan y la falta de motivación. (Sprang y Silman,2013 y Kuban y Steele, 2011).

\section{La enseñanza en línea: propincuidad digital y comunidad de indagación}

Se entiende por educación en línea la docencia llevada a cabo en un dispositivo digital que va a facilitar el aprendizaje (Clark y Mayer, 2016) y no es sinónimo de la educación a distancia tradicional. Gracias a la tecnología, se puede dar una presencialidad diferida en el tiempo y el espacio y, por ello, se requiere de una mayor interacción y colaboración entre los participantes. Se busca un equilibrio entre el aprendizaje autorregulado y el aprendizaje activo y colaborativo, que puede llegar a ser mayor y más intenso que en la educación presencial. En ningún caso se debe percibir como un producto de segunda clase (García-Peñalvo, 2020).

Por tanto, siguiendo una concepción socioconstructivista del aprendizaje, entendida como un proceso interactivo en el que las personas aprenden unas de otras (Bruner, 1999), el profesor tiene que centrar su

\footnotetext{
${ }^{2}$ Fuente: https://en.unesco.org/covid19/educationresponse

${ }^{3}$ Esto es contrario a lo indicado en la agenda de los Objetivos de Desarrollo del Milenio de la ONU en cuanto a la calidad educativa: "Garantizar una educación inclusiva y equitativa de calidad y promover oportunidades de aprendizaje permanente para todos". Fuente: https://www.agenda2030.gob.es/objetivos/objetivo4.htm
} 
atención en favorecer la conexión entre estudiantes y fomentar que esta se extienda más allá del grupo de clase. Esto no es posible sin un aprendizaje cooperativo que, desde que empezó la pandemia mundial, ha sido trasladado al formato digital.

La teoría de la propincuidad ("Theory of Electronic Propinquity"), formulada por Korzenny, ha resurgido en el último año con fuerza, debido al auge de interacciones educativas en entornos nuevos o canales de comunicación digitales. Según Korzenny (1978), pese a que normalmente la proximidad psicológica ha sido vinculada a la cercanía física, característica de la comunicación personal presencial, gracias a los canales de comunicación electrónicos también se puede experimentar un sentimiento de cercanía (electronic propinquity).

De acuerdo con Ina Blau et al. (basándose en Korzenny, 1978), la comunicación en entornos virtuales promueve un sentimiento de cercanía o propincuidad, que refuerza las interacciones interpersonales entre los usuarios. Seis factores la determinan: amplitud de banda o canal (diversidad de información y contenidos a transmitir), complejidad de la información, capacidad de direccionalidad mutua, habilidades comunicativas de los sujetos, reglas de la comunicación y número de canales de comunicación alternativos (otros canales como el chat, el correo electrónico, la videoconferencia, etc.). El estudio de las relaciones entre estos permite evaluar la naturaleza de la comunicación humana en los medios digitales. Para ello, Korzenny (1978, p. 4) defiende estos seis principios básicos:

1) A más amplitud de banda o canal, más proximidad.

2) A más complejidad de la información, menos proximidad.

3) A más direccionalidad mutua del canal, más proximidad.

4) A más habilidad comunicativa de los sujetos, más proximidad.

5) A más cantidad de reglas comunicativas, menos la proximidad.

6) A menos selección de canales de comunicación alternativos, más proximidad.

Por tanto, desde esta perspectiva, el aprendizaje electrónico crea una nueva ecología del aula, con un alto potencial interactivo, que ya ha transformado las formas de educación y aprendizaje en el siglo XXI, cuyo objetivo es "unir diversidad y cohesión en una ecología de la formación dinámica e intelectualmente estimulante" (Anderson y Garrison, 2005, p.20). La finalidad es buscar la formación de conexiones dentro de la comunidad, formada por alumnos y profesores, a través de las expresiones de propincuidad entre los participantes (Figura $\mathrm{n}$ ‥ 1). 
Figura 1: Actos de habla que más propician la propincuidad (Cruz et al, 2012)

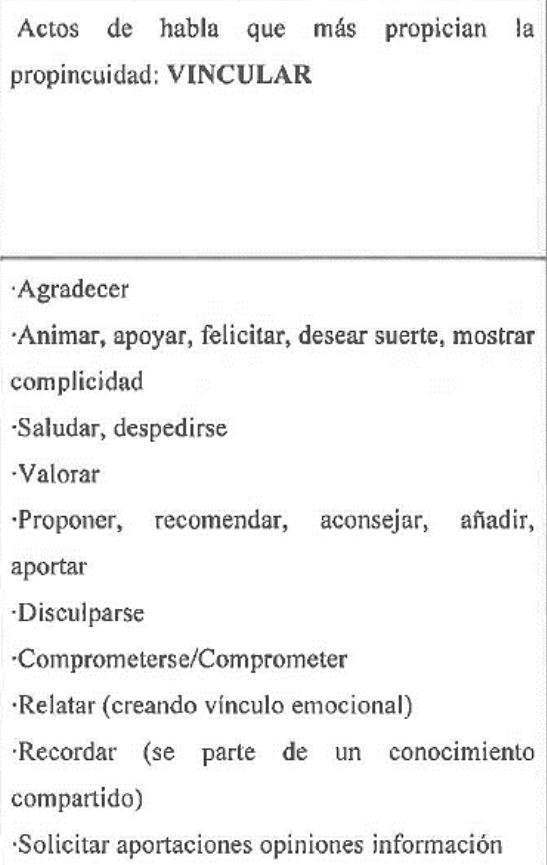

-Solicitar aportaciones opiniones información

También se contempla al grupo como una sociedad de aprendizaje que funciona con las características de una red social, viva y cambiante, en la que las expresiones de propincuidad y confianza resultan fundamentales para la generación y el flujo de conocimiento. Se trata del modelo de "comunidad de indagación" ("community of inquiry model"), propuesto por Anderson y Garrison (2005, p.44), quienes defienden que:

profesores y estudiantes que interactúan con el objetivo de facilitar, construir y validar la comprensión, y de desarrollar capacidades que conduzcan a continuar la formación en el futuro. Una comunidad de este tipo, fomenta simultáneamente la independencia cognitiva y la interdependencia social. Es la yuxtaposición de esos dos aspectos aparentemente contradictorios la que crea la chispa que enciende la experiencia educativa auténtica (Anderson y Garrison, 2005:44).

Además, establecen que, en las comunidades que se forman en los entornos digitales, el aprendizaje ocurre a través de la interrelación entre tres componentes centrales: presencia cognitiva, presencia docente y presencia social ${ }^{4}$.

\footnotetext{
${ }^{4}$ La presencia social, entendida como la habilidad de los participantes para "proyectarse a sí mismos social y emocionalmente, como personas reales (es decir, su personalidad plena dentro y fuera de la comunidad), mediante los medios de comunicación en uso". La presencia cognitiva es el elemento vital en el pensamiento crítico, el grado en que los sujetos participantes en la comunidad construyen significado a través de una comunicación sostenida con el objetivo de "posicionarse en el discurso para construir significado mediante la reflexión continua en una comunidad de investigación". La presencia docente,
} 
Figura 2: Modelo de la comunidad de indagación (Garrison, Anderson y Archer, 2000)

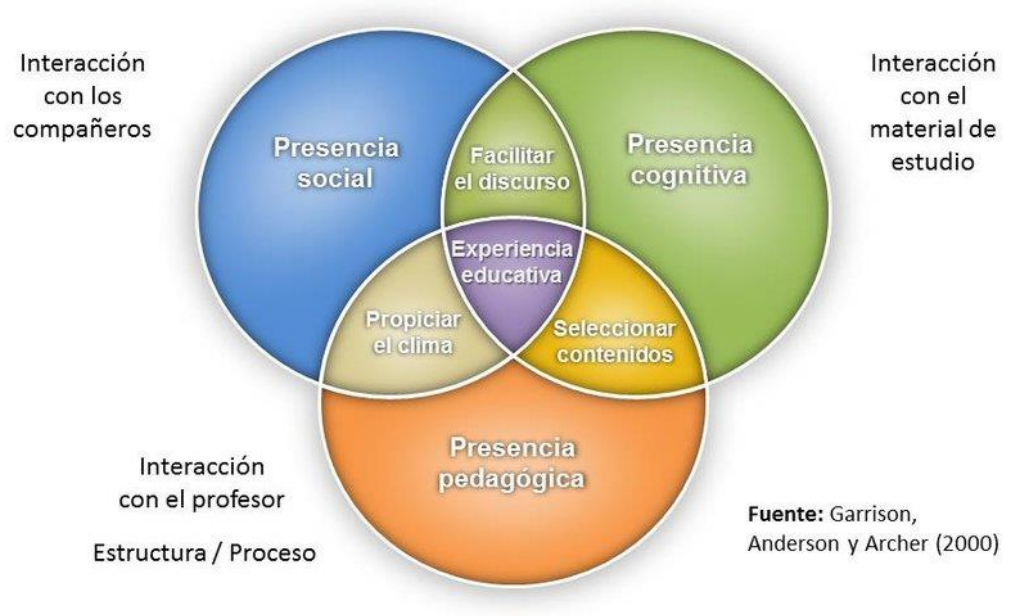

En esta figura, se ilustra cómo la conjunción de las tres presencias es significativa para la consecución de los objetivos de una comunidad de aprendizaje. Además, la unión de la presencia social y la cognitiva favorece el desarrollo de un discurso apropiado dentro del grupo; mientras que la presencia docente, centrada en la estructura y el proceso del aprendizaje, al coincidir con la presencia social, tiene repercusiones positivas para la experiencia educativa de los miembros de la agrupación.

Finalmente, estos autores proponen una serie de indicadores (palabras clave) para determinar la aparición de cada presencia en el discurso y sostienen que esta plantilla puede ser de gran utilidad para identificar las áreas docentes que deben ser atendidas para optimizar el discurso. Estas comunidades, para funcionar, necesitan un entorno adecuado a sus objetivos y necesidades. Cabe preguntarse qué características deben presentar estos entornos.

\section{Estudio de caso}

\subsection{Contexto}

En India, el primer ministro Narendra Modi anunció con apenas cuatro horas de antelación la imposición de uno de los confinamientos más estrictos del mundo que se alargó más de dos meses., que propició una transición forzosa a la enseñanza en línea.

Pese a ser el segundo país en cuanto a usuarios de internet (630 millones), la conectividad sigue siendo un problema, ya que la mayoría de

cuyo fin es desarrollar la presencia cognitiva para promover el aprendizaje, hace referencia a la actuación del profesor como organizador, facilitador y guía (papel que también puede ser desempeñado por lo estudiantes) para "obtener resultados educativos personalmente significativos y de valor docente" (Garrison y Anderson, 2005). 
los usuarios tienen una conexión móvil como principal fuente de internet ${ }^{5}$, en vez de una por fija.

En el caso concreto de la Universidad Jawaharlal Nehru (Nueva Delhi, India), donde hay alrededor de 8805 estudiantes, el 70\% vive en las residencias de la misma universidad en el campus situado en el sur de Nueva Delhi. De estos estudiantes residentes, alrededor de un $63 \%$ entran en las diferentes categorías de colectivos vulnerables definidas por el gobierno indio, ya sea por motivos económicos, físicos o religiosos (estos porcentajes se repiten en las diferentes facultades de la universidad). Es por ello que la universidad representa un ejemplo de la diversidad de la sociedad, además de ser de las pocas que ha estado entre los tres primeros puestos del ranking de las mejores universidades indias en los últimos años. Se trata, por tanto, de una institución referente en el ámbito de educación superior en el país. Para conocer con más detalle los estudiantes que formaron parte de este estudio, se llevó a cabo una encuesta en el Departamento de Filología española y Latinoamericana: solo el $50 \%$ de estos vivía en zonas urbanas, más del $80 \%$ usaba el teléfono móvil como dispositivo para asistir a las clases en línea ${ }^{6}$ y apenas un $25 \%$ disponía de una conexión Wi-Fi. De los que asisteron a las clases (el 90\%), menos de la mitad (el 47,7\%) lo hizo de manera regular y el $58 \%$ lo atribuyó a la mala conexión de internet de la que disponía.

Solo el $23,9 \%$ participaba activamente en clase. El resto, solo hablaba si el docente se lo pedía (el $65,7 \%$ ) y un $10,4 \%$ no lo hacía nunca. Otro dato relevante es el uso de la cámara - esencial para el lenguaje no verbal-: solo el 7,5\% encendía la cámara por iniciativa, si bien el 58,2\% lo hacía en caso de que el docente lo solicitara. Aquellos que nunca la encendían (el 33,8\%) lo atribuyeron a su mala conexión de internet (el $68,1 \%$ ), aunque más del $81 \%$ de ellos preferían que el docente la tuviera encendida.

De esta forma, la transición forzosa a la educación digital, causada por la situación de emergencia sociosanitaria, acentuó la brecha digital que ya existía, impidiendo que muchos estudiantes formaran parte de la comunidad de aprendizaje a la que pertenecían antes de que se declarara la pandemia mundial. Además, la capacidad de atención de los estudiantes, que antes de la situación de emergencia actual era de entre diez a quince minutos (Bradbury, 2016), resultó menoscabada por la incertidumbre y el

\footnotetext{
${ }^{5}$ En este país, el $90 \%$ de los estudiantes de educación superior tienen smartphones. A pesar de esto, los que viven en entornos rurales tienen una conexión a internet insuficiente: solo el $14,9 \%$ tiene internet por cable en sus casas y apenas un $4,4 \%$ posee un ordenador portátil. Sin embargo, en las zonas urbanas estos porcentajes difieren: el $42 \%$ tiene internet por cable en casa y el $23 \%$ cuenta con un ordenador portátil. Esto denota la gran disparidad que existe entre zonas urbanas y rurales ${ }^{\#}$ que, a su vez, repercute en la construcción de la identidad y la autoestima del alumnado, contrario al papel de lo que debería ser la educación (Bruner, 1999).

${ }^{6}$ Aunque el aprendizaje a través del móvil es ubicuo, también hay limitaciones debido a la funcionalidad inferior de los teléfonos en comparación con los ordenadores (D'Andrea et al., 2009).
} 
estrés causados por la pandemia de la COVID-19. Todo esto hizo que se planteara una profunda reflexión sobre el formato de enseñanza.

Por ello, inspirado en el modelo de los créditos ECTS ${ }^{7}$, gracias al que se le atribuye mayor grado de autonomía y responsabilidad sobre su formación al estudiante (Rue, 2007), se planteó una nueva distribución del tiempo de trabajo en el aula (digital) de manera síncrona -siguiendo el enfoque pedagógico de clase invertida ${ }^{8}-\mathrm{y}$ de trabajo autónomo de manera asíncrona, ya sea de manera individual o en grupo, a través de unos aplicativos (Padlet, Flipgrid, Telegram ${ }^{9}$ ) para permitir la interacción docenteestudiante y estudiante-estudiante, así como la accesibilidad de aquellos con recursos (tipo de conexión a internet y dispositivos) limitados o insuficientes. Todo esto tenía como objetivo buscar la formación de conexiones dentro de la comunidad de indagación (estudiantes y docentes), a través de las expresiones de propincuidad o cercanía entre los participantes, pese a la lejanía física.

\subsection{Participantes}

En este estudio formaron parte los estudiantes que están cursando el tercer año del grado de Filología española en JNU, en concreto la asignatura "SP 305 - Lengua española", cuya carga lectiva era de cuatro horas presenciales lectivas a la semana. En concreto, fueron 27 estudiantes (11 chicas y 16 chicos) de edades que oscilaban entre los 20 y los 23 años. En su mayoría su lengua materna era el hindi, si bien casi todos hablaban tres o cuatro lenguas oficiales del país. Su motivación para aprender español era de carácter profesional.

\footnotetext{
7 Sistema Europeo de Transferencia y Acumulación de Créditos (ECTS, por sus siglas en inglés), por el que se ha normalizado que la carga de trabajo estimada para cada asignatura a lo largo de un semestre sea de un $40 \%$ de presencialidad y el $60 \%$ restante de trabajo autónomo del estudiante. Se toma como referencia de medida de tiempo para cada asignatura que 1 crédito ECTS supone 25 horas de dedicación del estudiante, por lo que a una asignatura de 6 ECTS le corresponden 150 horas de dedicación. De éstas, 60 horas son presenciales y 90 horas de trabajo autónomo del estudiante (Espacio Europeo de Educación Superior, 2015).

${ }^{8}$ Enfoque metodológico en el que la instrucción directa se desplaza de la dimensión del aprendizaje grupal a la dimensión del aprendizaje dinámico e interactivo en el que el facilitador guía al alumnado en la aplicación de conceptos y en su implicación creativa del curso (Bergmann y Sams, 2014).

${ }^{9}$ Mientras que Padlet es una plataforma digital que permite crear murales colaborativos interactivos, así como la evaluación y laco-evaluación, Flipgrid ofrece la posibilidad de crear debates a través de vídeos. Telegram, además de ser un aplicativo de mensajería instantánea en el que no es necesario compartir el número de teléfono móvil personal, se pueden realizar encuestas entre los integrantes del grupo.
} 


\subsection{Procedimientos}

\section{Redistribución de la carga de trabajo}

Se presenta el siguiente formato de distribución de la carga de trabajo para la docencia en línea:

- el $40 \%$ del tiempo en clases de manera sincrónica (el docente y los aprendientes comparten el tiempo, pero no el espacio) siguiendo la metodología de la clase invertida;

- el $60 \%$ restante para trabajo autónomo en formato asincrónico (ni el docente ni los aprendientes comparten el espacio o el tiempo) a través de trabajos en grupo e individuales.

\section{Estructuración de cada tipo de sesiones: síncronas y asíncronas}

Figura 3. Tiempo utilizado en la "clase invertida" por taxonomía de Bloom (Tourón, Santiago y Díez, 2014)

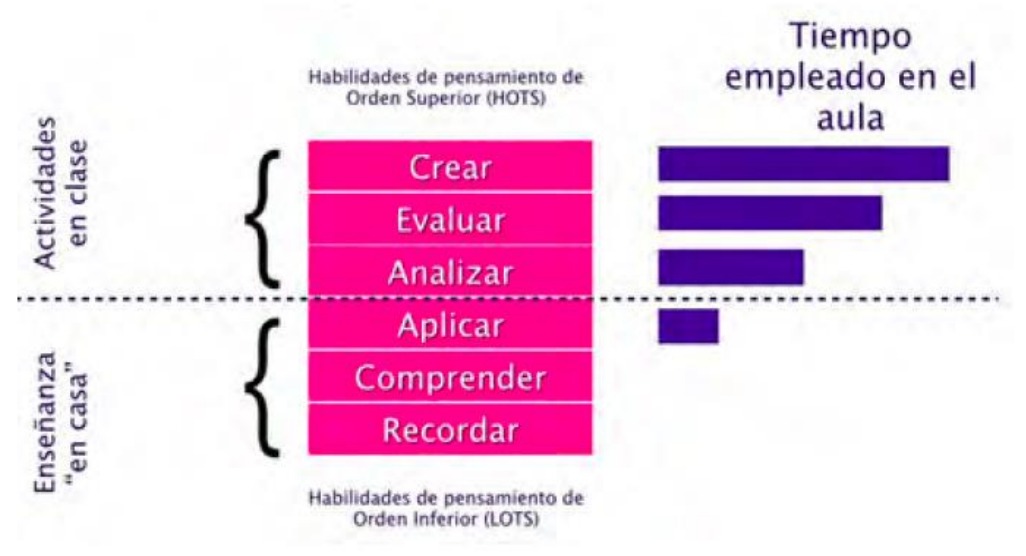


Figura 4: Actividades para "flipped classroom" antes, durante y después de la clase y su relación con los niveles de taxonomía de aprendizaje revisada de Bloom (Boslaugh, S. P., 2013). Fuente: theflippedclassroom.es

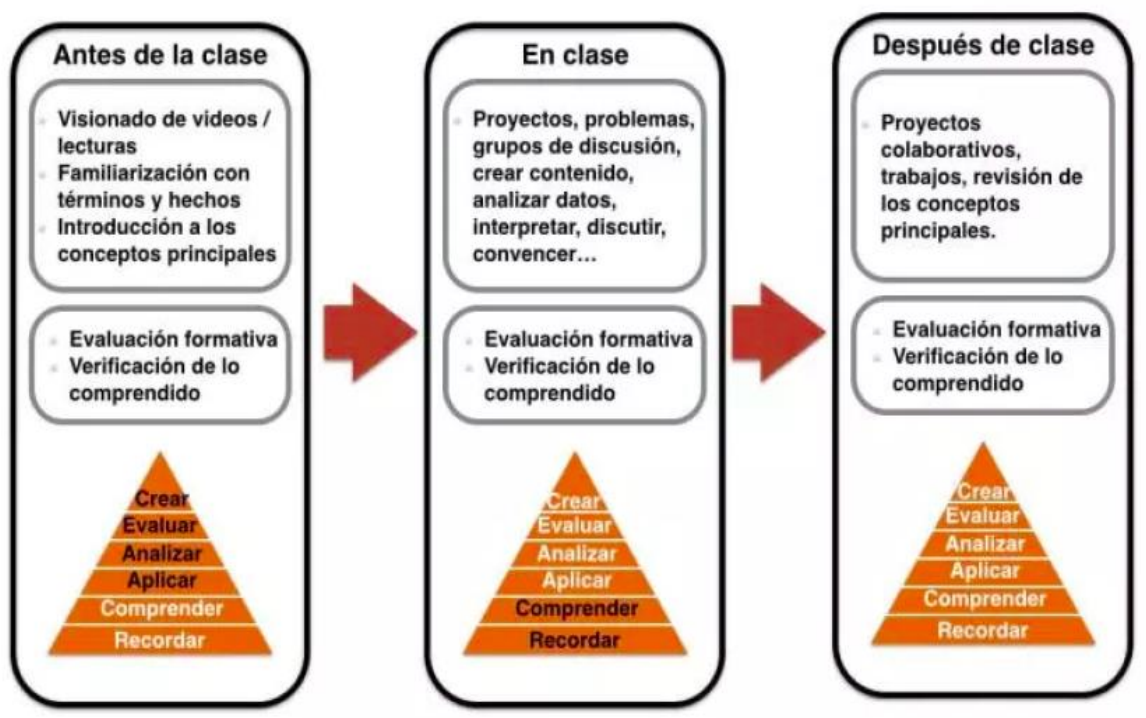

Las sesiones síncronas tuvieron lugar dos veces a la semana durante 50 minutos cada vez, es decir, el $40 \%$ del tiempo asignado por la universidad para esta asignatura. Cada sesión de este tipo se llevó a cabo desde el enfoque pedagógico de la clase invertida, a través del que se desarrollaron las habilidades de pensamiento de orden superior, descritas en la taxonomía de Bloom, es decir: "crear", "evaluar", "analizar "y, en menor medida, "aplicar" (Figuras 1 y 2). El manual empleado para estas sesiones fue Campus Sur B1, diseñado desde el enfoque metodológico de la clase invertida, cuyos temas eran actuales y de interés para estudiantes universitarios de español como lengua extranjera.

Tomando como referencia la estructura propuesta por Bergmann y Sams (2012), en nuestro contexto concreto, (Anejo I), así como los actos de habla que más propician la propincuidad digital, presentados anteriormente, se propuso la siguiente estructura: 
Tabla 1. Secuenciación de una sesión síncrona. Fuente: elaboración propia.

\begin{tabular}{|l|c||}
\hline \multicolumn{2}{|c||}{ Sesión síncrona } \\
\hline \multicolumn{1}{|c||}{ Actividad } & Tiempo \\
\hline $\begin{array}{l}\text { Calentamiento (música, preguntas } \\
\text { personales, conversaciones sobre temas no } \\
\text { relacionados con la temática de clase, } \\
\text { mensajes de fondo, etc. }{ }^{18} \text { ). }\end{array}$ & 10 minutos \\
\hline $\begin{array}{l}\text { Preguntas y respuestas sobre el material } \\
\text { preparado en casa para la clase. }\end{array}$ & 30 minutos \\
\hline $\begin{array}{l}\text { Actividad práctica y/o laboratorio guiado e } \\
\text { independiente basado en las habilidades de } \\
\text { pensamiento de orden superior. }\end{array}$ & 5 minutos \\
\hline $\begin{array}{l}\text { Reflexión final y cierre de la sesión con } \\
\text { imágenes y música de despedida. }\end{array}$ & \\
\hline
\end{tabular}

En cuanto a las sesiones asíncronas, estas tuvieron una carga de trabajo semanal de 140 minutos, es decir el 60\% en comparación con las sesiones síncronas. En estas, ni el docente ni los aprendientes compartieron el espacio o el tiempo, si bien, a través de diferentes aplicativos interactivos como Padlet o Flipgrid, y la mensajería instantánea (Telegram), aunque la conexión a internet no sea estable, se pudo mantener la interacción y se desarrolló esta cercanía entre docente-aprendientes y aprendientesaprendientes. Esta puede lograrse (Korzenny, 1978), gracias a que los miembros del mismo grupo de alumnos tenían objetivos compartidos (por ejemplo, comentar un vídeo de un tema relacionado con la temática de clase) y a que las respuestas se publicaron en un tiempo corto (máximo 48 horas). Todo ello facilitó la creación de enlaces referenciales internos que promovían la interacción.

En estas sesiones asincrónicas no se incluyó el tiempo que los estudiantes tenían que dedicar a la lectura de textos o al visionado de vídeos necesarios para las clases síncronas con el enfoque de la clase invertida (en la Figura 3, "antes de la clase"); fueron más bien una continuación de las sesiones síncronas en las que las habilidades de alto orden se seguían desarrollando, si bien de manera no simultánea (en la Figura 3, "Después de la clase" $)^{10}$.

\footnotetext{
${ }^{10}$ Durante todo el proceso, se llevó a cabo un seguimiento continuo y feed-forward de las actividades realizadas con el objetivo de detectar las fortalezas y debilidades del proceso de aprendizaje y poder establecer medidas correctoras o estrategias de mejora.
} 
Tabla 2: Comparativa del aula tradicional y el aula inversa.

\begin{tabular}{|l|c|l|l|}
\hline \multicolumn{2}{|c|}{ AULA TRADICIONAL } & \multicolumn{2}{c|}{ AULA INVERSA } \\
\hline \multicolumn{1}{|c|}{ Actividad } & \multicolumn{1}{c|}{ Tiempo } & \multicolumn{1}{c|}{ Actividad } & \multicolumn{1}{c|}{ Tiempo } \\
\hline Actividad de calentamiento & $5 \mathrm{~min}$ & Actividad de calentamiento & $5 \mathrm{~min}$ \\
\hline $\begin{array}{l}\text { Repaso anterior (Tareas de } \\
\text { casa) }\end{array}$ & $20 \mathrm{~min}$ & $\begin{array}{l}\text { Preguntas y respuestas so- } \\
\text { bre el video }\end{array}$ & $10 \mathrm{~min}$ \\
\hline $\begin{array}{l}\text { Lectura y trabajo de nuevo } \\
\text { contenido }\end{array}$ & $30-45 \mathrm{~min}$ & $\begin{array}{l}\text { Actividad práctica y/o labo- } \\
\text { ratorio guiado e indepen- } \\
\text { diente }\end{array}$ & $75 \mathrm{~min}$ \\
\hline $\begin{array}{l}\text { Actividad práctica y/o labo- } \\
\text { ratorio guiado e indepen- } \\
\text { diente }\end{array}$ & $20-35 \mathrm{~min}$ & \\
\hline
\end{tabular}

\subsection{Recogida de datos}

La recogida de datos se llevó a cabo en dos fases. En la primera, para evaluar el índice de propincuidad que se generó en las interacciones, tanto síncronas como asíncronas, los estudiantes que formaron parte del muestreo incidental tuvieron que rellenar una encuesta breve de cinco ítems, basada en la escala creada por Walther y Bazarova (2008) desde la perspectiva de la escala de Likert ${ }^{11}$, tanto al finalizar la sesión síncrona como las actividades de trabajo colaborativo en el formato asíncrono. Esto permitió contrastar el impacto que tenían los diferentes tipos de interacciones a la hora de propiciar la propincuidad.

Por otro lado, en la segunda fase, para poder emprender un análisis de los contenidos lingüísticos y paralingüísticos de las interacciones, los investigadores desarrollaron un modelo de indicadores (Tabla 3) para la medición de cada uno de los tres componentes (presencia cognitiva, presencia social y presencia docente). A partir de estos elementos y categorías, se elaboró una encuesta con 34 ítems traducida al español y validada por un grupo de especialistas (Ballesteros et al., 2019) de la Universidad Nacional de Educación a Distancia (UNED) en 2019. En esta versión, se habían incorporado tres preguntas abiertas (una para cada dimensión o presencia del modelo de comunidad de indagación) que aportaban información cualitativa para ayudar al docente a valorar la percepción de los estudiantes. Esta fue la que se usó en esta pesquisa. Los estudiantes rellenaron esta encuesta, una vez finalizado el estudio.

11 Escala de calificación cualitativa que se utiliza para cuestionar a una persona sobre su nivel de acuerdo o desacuerdo con una declaración. 
Tabla 3. Sistema de categorización del modelo de comunidad de indagación (Anderson, Archer y Garrison, 2000). Fuente: Gros y Silva (2006).

\begin{tabular}{|lll|}
\hline Elementos & Categorías & Indicadores (ejemplos) \\
\hline $\begin{array}{l}\text { Presencia } \\
\text { cognitiva }\end{array}$ & - Hecho desencadenante (Triggering Event) & - Sensación de perplejidad \\
& - Exploración (Exploration) & - Intercambio de información \\
& - Integration (Integration) & - Asociación de ideas \\
Presencia & - Dimensión afectiva (Emotional Expression) & - Aplicar nuevas ideas \\
social & - Comunnicación abierta (Open Communication) & - Expresar las emociones \\
& - Cohesión de grupo (Group Cohesión) & - Promover la cooperación \\
$\begin{array}{l}\text { Presencia } \\
\text { docente }\end{array}$ & - Diseño y organización (Instructional Management) & - Establecer el programa de \\
& - Promover la elaboración discursiva (Building & contenidos \\
& Understanfing) & - Construir el significado \\
& - Orientación explícita (Direct Instruction) & - Centrar el debate
\end{tabular}

Por último, con el objetivo de evaluar las percepciones sobre el entorno de aprendizaje invertido en el aula, se utilizó el cuestionario diseñado por Gilboy et al. (2015) al finalizar el periodo de estudio: cinco ítems, también con una escala Likert, con cinco opciones (muy de acuerdo, de acuerdo, neutral, en desacuerdo o muy en desacuerdo).

\section{Análisis comparativo de los datos obtenidos y conclusiones derivadas}

En primer lugar, es importante mencionar el nivel de participación en este estudio: la asistencia a clase era voluntaria y no tenía repercusiones académicas según la normativa vigente de la universidad. Participó el 70\% de los estudiantes, de los cuales el $75 \%$ fueron mujeres y el $25 \%$ hombres. De este $75 \%$, el $40 \%$ participó tanto en las sesiones síncronas como en las asíncronas y el $60 \%$ restante solo en las asíncronas.

A continuación, se procedió a comparar el grado de propincuidad al que los estudiantes llegaron en las sesiones síncronas y las asíncronas. Siguiendo la escala de Walther y Bazarova (2008), se pudo observar una inclinación en las respuestas hacia los ítems que se identifican con una cercanía o propincuidad desde la primera sesión hasta la última. En las siguientes tablas se detallan los resultados de las sesiones síncronas (Tabla 4) y asíncronas (Tabla 5). 
Tabla 4. Evaluación de la propincuidad en las sesiones síncronas. Fuente: Elaboración propia

\begin{tabular}{|c|c|c|}
\hline \multicolumn{3}{|c|}{ Evaluación de la propincuidad en las sesiones síncronas } \\
\hline Item & Primera encuesta & Última encuesta \\
\hline Distante - Cercano & $\begin{array}{l}\text { El } 50 \% \text { marcó la opción «un poco } \\
\text { cercano»; el resto, optó por } \\
\text { «neutro» (33\%) y «un poco } \\
\text { distante }(16 \%) » .\end{array}$ & $\begin{array}{l}\text { El } 50 \% \text { se mostró neutral y el resto } \\
(40 \%) \text { un poco cercano y }(10 \%) \\
\text { cercano. }\end{array}$ \\
\hline Cerca - Lejos & $\begin{array}{l}\text { Más de la mitad }(66 \%) \text { se inclinó } \\
\text { por la opción «neutro»; el resto se } \\
\text { decantó hacia «un poco cerca». }\end{array}$ & $\begin{array}{l}\text { La mitad marcó «un poco cerca», } \\
\text { el } 40 \% \text { «neutro» y el } 10 \% \text { «un poco } \\
\text { lejos». }\end{array}$ \\
\hline Juntos - Separados & $\begin{array}{l}\text { El } 50 \% \text { se mostró neutral, el resto } \\
\text { a partes iguales indicó «un poco } \\
\text { juntos», «un poco separados» y } \\
\text { «separados». }\end{array}$ & $\begin{array}{l}\text { El } 40 \% \text { se identificó con la opción } \\
\text { «un poco juntos»; el resto a partes } \\
\text { iguales seleccionó las opciones } \\
\text { «juntos»y «neutro»., }\end{array}$ \\
\hline Próximo - Remoto & $\begin{array}{l}\text { Dos terceras partes }(66,7 \%) \text { marcó } \\
\text { la opción "un poco próximo"; el } \\
\text { resto a partes iguales seleccionó } \\
\text { las opciones "próximo» y } \\
\text { «neutro». }\end{array}$ & $\begin{array}{l}\text { La mitad se mostró neutral; el } 40 \% \\
\text { un poco próxima y el resto }(10 \%) \\
\text { un poco remoto. }\end{array}$ \\
\hline Desconectado - Conectado & $\begin{array}{l}\text { La mitad de los estudiantes se } \\
\text { sintió un poco conectada al resto, } \\
\text { el } 30 \% \text { conectados y el } 20 \% \\
\text { neutrales. }\end{array}$ & $\begin{array}{l}\text { La mitad de los alumnos se sintió } \\
\text { conectada, el } 30 \% \text { un poco } \\
\text { conectada y el } 20 \% \text { neutral. }\end{array}$ \\
\hline
\end{tabular}

Tabla 5. Evaluación de la propincuidad en las sesiones asíncronas. Fuente: Elaboración propia

\begin{tabular}{|c|c|c|}
\hline \multicolumn{3}{|c|}{ Evaluación de las sesiones asíncronas } \\
\hline Ítem & Primera encuesta & Última encuesta \\
\hline Distante - Cercano & $\begin{array}{l}\text { Tres cuartas partes se mostraron } \\
\text { neutrales; el resto, a partes } \\
\text { iguales, «distantes» y "un poco } \\
\text { cercanas». }\end{array}$ & $\begin{array}{l}\text { El } 60 \% \text { se identificó con «un poco } \\
\text { cercano», el } 20 \% \text { con «cercano» y } \\
\text { el } 20 \% \text { con «neutral» }\end{array}$ \\
\hline Cerca - Lejos & $\begin{array}{l}\text { El } 87,5 \% \text { seleccionó la opción } \\
\text { «neutro»; el } 12,5 \% \text { «un poco } \\
\text { lejos». }\end{array}$ & $\begin{array}{l}\text { El } 40 \% \text { marcó la respuesta «un } \\
\text { poco cerca»; el } 30 \% \text { «cerca» y el } \\
20 \% \text { «neutro». }\end{array}$ \\
\hline Juntos - Separados & $\begin{array}{l}\text { El } 62,5 \% \text { se identificaron con la } \\
\text { opción «un poco separados», y el } \\
\text { resto a partes iguales con «un } \\
\text { poco juntos», «neutro» y } \\
\text { «separados». }\end{array}$ & $\begin{array}{l}\text { El } 40 \% \text { seleccionó la opción } \\
\text { «juntos», el otro } 40 \% \text { «un poco } \\
\text { juntos»y el resto «neutro». }\end{array}$ \\
\hline Próximo - Remoto & $\begin{array}{l}\text { El } 75 \% \text { marcó la opción «neutro» y } \\
\text { el } 25 \% \text { «un poco juntos». }\end{array}$ & $\begin{array}{l}\text { El } 20 \% \text { se identificó con la opción } \\
\text { «próximo»; el resto, a partes } \\
\text { iguales ( } 40 \% \text { cada una), con las } \\
\text { opciones «un poco próximo»y } \\
\text { «neutro». }\end{array}$ \\
\hline Desconectado - Conectado & $\begin{array}{l}\text { El } 62,5 \% \text { se sintió un poco } \\
\text { conectado, el } 25 \% \text { conectado y el } \\
12,5 \% \text { desconectado. }\end{array}$ & $\begin{array}{l}\text { El } 60 \% \text { se sintió un poco } \\
\text { conectado, el } 20 \% \text { neutral y el otro } \\
20 \% \text { conectado. }\end{array}$ \\
\hline
\end{tabular}


Tras observar esta tabla, cabe destacar los resultados del nivel de propincuidad digital que manifestó el alumnado en las clases asíncronas. Pese a que el mayor porcentaje no correspondía al valor que indicaba la propincuidad más alta (es decir, "conectado", "próximo" o "juntos"), la tendencia después de la última sesión fue hacia los valores que apuntaban a una mayor propincuidad, en comparación a la primera sesión asíncrona, como se puede ver la tabla número cuatro. También hubo una inclinación similar en las sesiones síncronas. No obstante, este fenómeno no sorprende especialmente porque, como se ha visto antes, la presencialidad, si bien en este caso sea digital, ya propicia esa propincuidad. Este fenómeno demostró que aquellos alumnos que solo asistieron a las sesiones asíncronas pudieron experimentar esa cercanía digital, pese a no compartir ni el espacio ni el tiempo con sus compañeros de clase.

Además, esta tendencia se vio manifestada en los actos de habla que más favorecían esa cercanía (Figura 1) y que fueron empleados por los estudiantes (se detallan los mismos más abajo en la tabla número siete). Se pudo observar que era en los mensajes que pertenecían a estos actos de habla donde más interacción se logró: aquellos comprendidos dentro de la presencia social, en concreto los que se refieren a intervenciones vinculadas con comportamientos socio-emocionales (Anejo IV). Para ver el nivel en que las presencias estaban más representadas, se tomó como referente la siguiente tabla:

Tabla 6. Nivel de representación de las presencias del modelo de indagación según el cuestionario traducido al español de la comunidad de indagación (Ballesteros et al. 2019). Fuente: Elaboración propia

\begin{tabular}{|c|c|c|}
\hline $\begin{array}{l}\text { Nivel de representación de cada } \\
\text { presencia }\end{array}$ & Porcentaje & Ítems \\
\hline Alta & $\begin{array}{l}\text { Más del } 80 \% \text { de las respuestas se } \\
\text { sitúan entre utotalmente de } \\
\text { acuerdo" o «de acuerdo». }\end{array}$ & $\begin{array}{l}\text { Presencia docente: } 1,2,3,4,5,6 \text {, } \\
7,8,9,10,11,12 \text { Presencia social: } \\
17,18,19,22 \\
\text { Presencia cognitiva: } 24,25,33,34\end{array}$ \\
\hline Mediana & $\begin{array}{l}\text { Más del } 60 \% \text { de respuestas se } \\
\text { sitúan entre «totalmente de } \\
\text { acuerdo» o «de acuerdo». }\end{array}$ & $\begin{array}{l}\text { Presencia docente: } 13 \text { Presencia } \\
\text { social: } 14,15,16,20,21 \\
\text { Presencia cognitiva: } 26,27,28 \text {, } \\
29,30,31\end{array}$ \\
\hline Baja & $\begin{array}{l}\text { Menos del } 60 \% \text { de las respuestas } \\
\text { se sitúan entre } \text { atotalmente de } \\
\text { acuerdon }\end{array}$ & $\begin{array}{l}\text { Presencia docente: } 0 \\
\text { Presencia social: } 0 \\
\text { Presencia cognitiva: } 23,32\end{array}$ \\
\hline
\end{tabular}

Según los resultados de la tabla número cinco, se pudo observar que la presencia docente era la que tenía un nivel mayor de representación (un $92,3 \%$ ) en la categoría "alta", seguida por la social $(44,5 \%)$ y la cognitiva (33, $3 \%)$. Pese a eso, el $50 \%$ de la presencia cognitiva estuvo representada dentro de la categoría "mediana", el 55,5\% de la social y el 7,7\% de la docente. Por 
último, el $16,6 \%$ de la presencia cognitiva estuvo representada en la categoría "baja".

Pasando al análisis de las interacciones y siguiendo el sistema de categorización del modelo de comunidad de indagación (Anderson, Archer y Garrison, 2000) expuesto en la tabla número dos, se elaboró la Tabla 6 para analizar los mensajes en cada una de las categorías de indicadores de las presencias de las comunidades de indagación.

Los mensajes analizados fueron extraídos de las interacciones escritas (para poder llevar un recuento detallado) que dieron de manera asíncrona en los aplicativos Padlet, Google Classroom y Telegram ${ }^{12}$. En estas interacciones, entre un $70 \%$ y un $85 \%$ de los estudiantes intervinieron, frente a las interacciones orales a través del aplicativo Flipgrid, que, difícilmente, estimularon el diálogo en los debates: tan solo el $20 \%$ de los estudiantes participó en estas interacciones ${ }^{13}$.

Tabla 7. Mensajes en cada una de las categorías de indicadores de las presencias de las comunidades de indagación. Elaboración propia en base a las categorías propuestas por Garrison \& Anderson (2005)

\begin{tabular}{|c|c|c|c|}
\hline Presencia & Categoría & №. & \\
\hline \multirow{3}{*}{$\begin{array}{l}\text { PRESENCIA } \\
\text { SOCIAL }\end{array}$} & Comunicación afectiva & 41 & $19,2 \%$ \\
\hline & Comunicación abierta & 28 & $13,3 \%$ \\
\hline & Comunicación para la cohesión del grupo & 21 & $10 \%$ \\
\hline \multirow{4}{*}{ PRESENCIA COGNITIVA } & Activación o hecho desencadenante & 4 & $1,8 \%$ \\
\hline & Exploración & 13 & $6,2 \%$ \\
\hline & Integración & 8 & $3,7 \%$ \\
\hline & Resolución de problemas & 10 & $4,7 \%$ \\
\hline \multirow{4}{*}{ PRESENCIA DOCENTE } & Diseño y organización de enseñanza & 21 & $10 \%$ \\
\hline & $\begin{array}{l}\text { Intervenciones del docente para la facilitación } \\
\text { del discurso }\end{array}$ & 42 & $20,1 \%$ \\
\hline & Enseñanza directa & 24 & $11 \%$ \\
\hline & Número de mensajes totales & 212 & \\
\hline
\end{tabular}

\footnotetext{
12 En estos aplicativos en los que se desarrolla la comunidad se produjeron también otro tipo de respuestas no textuales que se originaron a través de los botones de interacción conocido como "me gusta". Estas interacciones tampoco se tuvieron en cuenta para este estudio.

13 En los comentarios del cuestionario "Comunidad de indagación", en la sección "Dimensión social", el $60 \%$ de los encuestados se mostró en contra de crear debates o conversaciones que requirieran la grabación de un vídeo semanalmente porque se sentían demasiado expuestos $y$, por tanto, el filtro afectivo estaba demasiado elevado. Como consecuencia, sus habilidades de expresión oral se vieron impactadas negativamente.
} 
En esta tabla se pudo constatar que es la presencia social la que más protagonismo adquirió en las interacciones entre los estudiantes, a pesar de que en el cuestionario "Comunidad de indagación" aquella con un nivel más alto de representación fuera la docente.

Esto no debe sorprender si se tiene en cuenta el contexto en el que se ha llevado a cabo el estudio. En India, por motivos culturales, el sistema educativo todavía percibe al docente como la única fuente de transmisión del conocimiento ${ }^{14}$, en vez de un facilitador o un guía como es el caso del modelo de la comunidad de indagación o del socioconstructivismo. Por eso, la presencia con menos representación fue la cognitiva, si bien no es del todo cierto, ya que la social, puesto que de manera indirecta tiene la función de mantener y reforzar la presencia cognitiva, se facilitaron los procesos de pensamiento crítico y unos niveles altos de presencia social. Esto, junto con altos grados de compromiso y participación, favoreció interacciones destinadas a desarrollar un entorno de aprendizaje cooperativo.

Por último, en cuanto al enfoque pedagógico empleado en las clases síncronas, es decir, la clase invertida, los estudiantes manifestaron lo siguiente:

- El 44,4\% de los estudiantes estuvo de acuerdo en que era una buena idea ver el vídeo antes de clase.

- El 33,3\% de los encuestados mostró neutral en relación a la metodología de este enfoque y el 22,2\% prefiró este enfoque.

- A dos terceras partes los vídeos y otros recursos les permitió aprender el material de estudio más eficazmente que hacer lecturas en solitario.

- Más de la mitad afirmó que había aprendido más con el enfoque del aula invertida que con el tradicional.

- Dos terceras partes se sintieron conectadas a pesar de que el profesor no estuviera físicamente presente durante los vídeos o actividades virtuales.

\section{Conclusiones}

Teniendo en cuenta los datos mencionados en el apartado anterior, se concluyó que, gracias a este estudio, se pudo demostrar que, aun estando en una situación de enseñanza de emergencia, sí se pueden implementar herramientas que faciliten la interacción en la adquisición del español como lengua extranjera. No obstante, hay que tener en cuenta algunos inconvenientes a la hora de llevar a cabo un enfoque pedagógico disruptivo.

\footnotetext{
14 “Como dijo Vivekananda: 'Es imposible adquirir el conocimiento a menos que se transmita de un discípulo a otro a través de una sucesión apostólica, a menos que llegue por medio de la misericordia del Maestro y directamente de su boca'"' (Bharati, 2005; Thapan, 2006).
} 
En primer lugar, la participación por parte del estudiantado fue menor de la esperada, tanto en las sesiones síncronas y asíncronas. Esto puso de relieve la mencionada brecha digital, así como la apatía telemática que muchos estudiantes sufrían tras más de un año de incertidumbre y enseñanza en línea forzada. También cabe mencionar aquí las diferencias culturales que hay entre el sistema tradicional de enseñanza indio (basado en la repetición de conceptos, sin apenas espacio para la creatividad y con el docente con un papel central en el aula) y lo recogido en el MCER diseñado por y para aprendientes europeos - , donde uno de los elementos clave en los que se hace hincapié es en la autonomía del aprendizaje, así como en los dictámenes del modelo de Comunidad de indagación, del enfoque pedagógico del aula invertida y del modelo de los créditos ECTS.

En segundo lugar, la falta de recursos sigue siendo una de las razones por las que muchos estudiantes de universidades públicas de un país como India no pueden acceder a la educación en línea y lo seguirá siendo hasta que termine esta situación de crisis a la que la comunidad educativa se ha visto forzada a transitar sin preparación alguna. Otra limitación que se debe tener en cuenta es la temporal, determinada porque el inicio del semestre fue retrasado por la pandemia. Para este tipo de estudio, se tuvo que diseñar un plan de clase un tanto encorsetado que pudo haber afectado la socialización entre los estudiantes y dejó en el segundo plano importantes cuestiones relacionadas con el desarrollo de estrategias de autoaprendizaje.

Pese todo esto, las conexiones dentro de la comunidad formada por el alumnado y los docentes se han visto reforzadas a través de expresiones de propincuidad asíncronas en diferentes aplicativos, aun cuando los estudiantes nunca han asistido a las sesiones síncronas. Los resultados han dejado claro que la presencia social es la predominante, seguida por la docente y la cognitiva.

No nos extraña que sea así, ya que la función predominante del lenguaje es la social (un ejemplo de ello son los comentarios realizados por los estudiantes en publicaciones de Padlet que no generaron mucha presencia cognitiva, aunque sí mucha social). En definitiva, los participantes establecieron vínculos con el resto que les permitió, además de interactuar en español, establecer un clima de confianza adecuado para el flujo de conocimiento creando un sistema ecológico de trabajo colaborativo que funcionara como una red social, es decir, viva y cambiante.

\section{BiBLIOGRAFÍA}

Alawamleh, M., Al-Twait, L. M. y Al-Saht, G. R. (2020): "The effect of online learning on communication between instructors and students during Covid-19 pandemic". Asian Education and Development Studies. Volumen 9, pp. 3-10. https://doi.org/10.1108/AEDS-06-2020-0131

ARCE FERNÁNDEZ, I. (2017): Los blogs como espacio de interacción más allá del aula presencial. Memoria de Máster en Lingüística Aplicada a la enseñanza del español como lengua extranjera. Universidad Nebrija. 
https://blogespaciointeraccion.files.wordpress.com/2013/10/tfm is aura arcefernandez blogs.pdf

ARETIO, L. G. (2017): “Educación a distancia y virtual: calidad, disrupción, aprendizajes adaptativo y móvil". RIED. Revista Iberoamericana de Educación a Distancia, 20(2), pp. 9-25.

Ballesteros Velázquez, B., Gil-Jaurena, I. y Morentin Encina, J. (2019): "Validación de la versión en castellano del cuestionario 'Community of Inquiry". Revista De Educación a Distancia (RED), 19(59), pp. 2426. https://doi.org/10.6018/red/59/04

Bradbury, N. A. (2016): "Attention span during lectures: 8 seconds, 10 minutes, or more?". Advances in physiology education, 40(4), pp. 509-513.

Belmar, M. C. y Escamilla, E. G. (2012): "Carga de trabajo autónomo del estudiante: tiempo de dedicación real y estimación de los docentes". En Álvarez, J., Tortosa, M., Pellín, N. (coords.): X Jornades de Xarxes d'Investigació en Docència Universitària: la participació $i$ el compromís de la comunitat universitària Instituto de Ciencias de la Educación. pp. 595-607.

BRINKMANN, S. (2016): The role of teachers' beliefs in the implementation of learner-centred education in India (Doctoral dissertation, UCL (University College London).

Di Pietro, G., BiAgI, F., Costa, P., KARPiński, Z. y MazzA, J. (2020): The likely impact of COVID-19 on education: Reflections based on the existing literature and recent international datasets (Vol. 30275). Publications Office of the European Union.

García-PeÑAlVo, F. J. (2020): "Modelo de referencia para la enseñanza no presencial en universidades presenciales". Campus Virtuales, 9 (1), pp. 41-56.

García-Valcárcel, A., Hernández Martín, A. y Recamán Payo, A. (2012): “La metodología del aprendizaje colaborativo a través de las TIC: una aproximación a las opiniones de profesores y alumnos". Revista Complutense de Educación, Vol. 23, 1, Universidad de Salamanca, pp. 161-188.

González-Miy, D., Herrera Díaz, L. E. y Díaz Camacho, J. E. (2015). “El modelo de Comunidad de Indagación". En EsquiveL, I. (coord.). Los Modelos Tecno-Educativos, México, pp. 67-75.

Gruber, A. y BAUER, E. (2020): "Fostering interaction in synchronous online class sessions with foreign language learners". En FERDIg, R.E., Baumgartner, E., Hartshorne, R., Kaplan-Rakowski, R. \& Mouza, C. (coord.): Teaching, Technology, and Teacher Education during the COVID-19 Pandemic: Stories from the Field, pp. 175-178. Association for the Advancement of Computing in Education (AACE).

HALL, J. K. (2010): "Interaction as method and result of language learning". Language Teaching, 43(2), pp. 202-215.

InStituto CeRVANTES. (2011). Marco común europeo de referencia para las lenguas: aprendizaje, enseñanza y evaluación. Instituto Cervantes. 
JAUREGI, K. (2013): La interacción, elemento clave en el proceso de aprendizaje de E/LE. Borrell, S. (coord.), Actas del XXIII Congreso Internacional ASELE. Girona: Asociación para la Enseñanza del Español como Lengua Extranjera, pp. 5-16.

Khan, S. y MohaKUd, L. (2020): Covid-19 and Digital Divide in Higher Education: Exploring the Indian Scenario, The Human Rights Issues in India, volumen 1, capítulo IV, pp.353-372. Adawal, S. y Behera, B.

KorzenNy, F. (1978): "A theory of electronic propinquity: Mediated communication in organizations". Communication Research, 5(1), pp. 3-24.

MARTíneZ FloR, A. (1999): Does language learners benefit from classroom interaction? Fòrum de Recerca, no. 5, 1999-2000, pp. 2-5.

Moreira, J., Ferreira, A. y AlmeidA, A. (2013): "Comparing communities of inquiry of Portuguese higher education students: One for all or one for each?", Open Praxis, 5(2), pp. 165-178.

NONELLI, O. E. (2011): Análisis de la interacción en un curso de ELE a distancia (Tesis doctoral), Barcelona, Universidad de Barcelona.

Pérez, Á. G., Rodríguez, A. M. P., Merino, C. S. y SÁez, F. T. (2020): “Tecnología para la Enseñanza y el Aprendizaje de Lenguas Extranjeras. La Enseñanza de lenguas Asistida por Ordenador. Pasado, presente y futuro". Pragmalingüística, (28), pp. 238-254.

Pérez-López, E., Atochero, A. V. y Rivero, S. C. (2021): “Educación a distancia en tiempos de COVID-19: Análisis desde la perspectiva de los estudiantes universitarios". Revista Iberoamericana de Educación a Distancia, 24(1), pp. 331-350.

Piñol, M. C., Buyse, K., Argüello, V. G. y ThKahara, N. (2011): “¿Qué queremos de la Red y para qué? Nuevas perspectivas en el uso de la Red en la enseñanza de ELE”. Hernández González, C., Carrasco Santana, A. y Álvarez Ramos, E. La Red y sus aplicaciones en la enseñanzaaprendizaje del español como lengua extranjera, Valladolid, Asociación para la Enseñanza del Español como Lengua Extranjera, pp. 31-60.

RAPanta, C., Botturi, L., Goodyear, P., GuÀrdia, L. y Koole, M. (2020): “Online university teaching during and after the Covid-19 crisis: Refocusing teacher presence and learning activity". Postdigital Science and Education, 2(3), pp. 923-945.

Thoms, J. J. (2012): "Classroom Discourse in Foreign Language Classrooms: A Review of the Literature". Foreign Language Annals, 45(S1), pp. 827. https://doi.org/10.111/j.1944-9720.2012.01177.x.FOREIGN 